\title{
Genital Primary Herpetic Infection With Concurrent Hepatitis in an Infant
}

Jae Woo Ahn, MD; Jung Woo Lee, MD; Hae Jun Song, MD, PhD; Chil Hwan Oh, MD, PhD; Jiehyun Jeon, MD, PhD

\section{PRACTICE POINTS}

- Parents with a history of herpes simplex virus (HSV) need to be educated before the baby is born to be careful about direct skin contact with the child to prevent the spread of HSV infection.

- Although systemic involvement is not typical, additional tests to rule out internal organ involvement may be required, especially in children.

To the Editor:

Cutaneous herpes simplex virus (HSV) infection generally involves mucocutaneous junctions, but virtually any area of the skin can be affected. ${ }^{1}$ When the genital area of adult patients is affected, the disease usually is sexually transmitted and mainly caused by HSV-2. In infants, genital primary herpetic infection is rare and more commonly is caused by HSV-1 than by HSV-2. We report a rare case of genital primary herpetic infection with concurrent hepatitis in an infant.

An 8-month-old infant with no underlying medical problems, including atopic dermatitis, was referred for erythematous grouped vesicles with erosions on the perianal area of 4 days' duration (Figure). The skin color appeared normal, not icterus. She also had a fever (temperature, $37.9^{\circ} \mathrm{C}$ ), and her urination pattern had changed from normal to frequent leakage, possibly owing to pain related to the eroded lesions. Physical examination did not reveal palpable inguinal lymph nodes. The oral mucosa was not involved. The patient's father had a history of recurrent herpetic infection on both the perioral and perianal areas.

A Tzanck smear revealed giant multinucleated cells with multiple inflammatory cells. Laboratory tests revealed marked leukocytosis, elevated liver enzymes (aspartate aminotransferase, 141 IU/L [reference range, $15 \mathrm{IU} / \mathrm{L}-60 \mathrm{IU} / \mathrm{L}]$; alanine aminotransferase, $422 \mathrm{IU} / \mathrm{L}$ [reference range, $13 \mathrm{IU} / \mathrm{L}-45 \mathrm{IU} / \mathrm{L}]$ ), and was positive for herpes simplex viral IgM but negative for herpes simplex viral IgG. A viral culture also demonstrated the growth of HSV. An abdominal ultrasound was normal. Based on the cutaneous and laboratory findings, genital

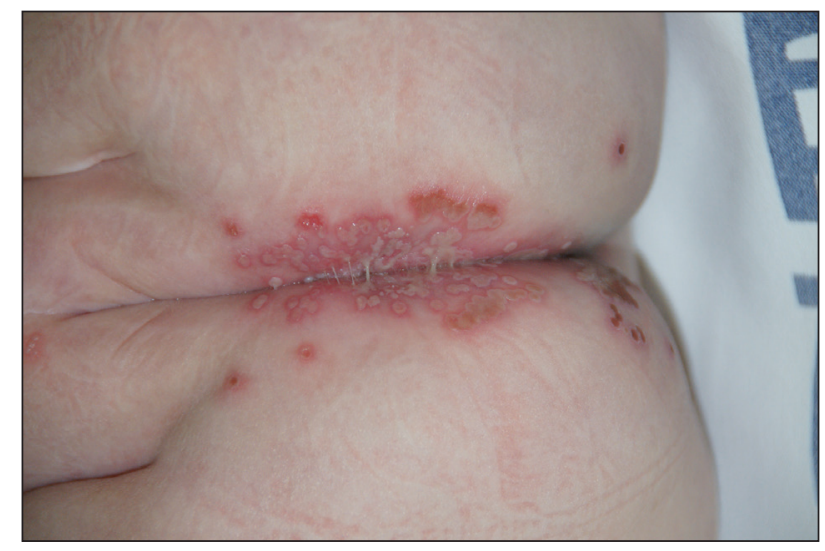

Genital primary herpetic infection. Multiple erythematous grouped vesicles with erosions in the perianal area of an infant. 
primary herpetic infection with concurrent hepatitis was diagnosed. Intravenous acyclovir $50 \mathrm{mg}$ was administered 3 times daily for 7 days, and a wet dressing with topical mupirocin was employed daily until the skin lesions healed. The fever subsided soon after starting treatment. The liver enzyme counts decreased gradually in serial follow-up (aspartate aminotransferase, $75 \mathrm{IU} / \mathrm{L}$; alanine aminotransferase, $70 \mathrm{IU} / \mathrm{L}$ ).

Primary herpetic infection usually is asymptomatic, but when symptoms do occur, it is characterized by the sudden onset of painful vesicle clusters over erythematous edematous skin. Lesions can be associated with fever and malaise and may involve the perineum. Urinary symptoms may occur. The average age of onset ranges from 6 months to 4 years. The virus commonly is transmitted by asymptomatic carriers. Autoinoculation from concomitant oral primary herpetic infection or individuals with active herpetic infection is one possible route of transmission. In our patient, we assumed that she acquired the virus from her father during close contact. A diagnosis can be made clinically using direct methods including culture, Tzanck smear, or polymerase chain reaction, or indirect methods such as serologic tests. ${ }^{2}$

Hepatitis secondary to HSV infection is rare, especially in immunocompetent patients. It occurs during primary infection and rarely during recurrent infection with or without concomitant skin lesions. ${ }^{3}$ Symptoms include fever, anorexia, nausea, vomiting, abdominal pain, leukopenia, coagulopathy, and marked elevation of serum transaminase levels without jaundice. Based on our patient's elevated liver enzyme levels and virological evidence of acute primary HSV infection, a lack of evidence of other hepatic viral infections, and the presence of herpes simplex viremia, we concluded that this infant had viral hepatitis as a part of the clinical presentation of primary HSV infection. We did not perform a direct liver biopsy considering her age and accompanying risks. ${ }^{4}$

Primary herpetic infection usually has a benign course and a short duration. In children, the prognosis depends on underlying immunologic status, not a particular type of HSV. In children with atopic dermatitis, primary herpetic infection tends to occur earlier and is more severe. Early treatment with acyclovir is effective; intravenous treatment is not required unless local complications or systemic involvement are present. Long-term follow-up is recommended because of the possibility of recurrence.

Although the possibility of systemic involvement including hepatitis due to HSV infection is low, awareness among dermatologists about primary herpetic infection and its possible complications would be helpful in the diagnosis and treatment, especially for atypical or extensive cases.

\section{REFERENCES}

1. Jenson HB, Shapiro ED. Primary herpes simplex virus infection of a diaper rash. Pediatr Infect Dis J. 1987;6:1136-1138.

2. Batalla A, Flórez A, Dávila P, et al. Genital primary herpes simplex infection in a 5-month-old infant. Dermatol Online J. 2011;17:8.

3. Norvell JP, Blei AT, Jovanovic BD, et al. Herpes simplex virus hepatitis: an analysis of the published literature and institutional cases. Liver Transpl. 2007;13:1428-1434.

4. Chen CK, Wu SH, Huang YC. Herpetic gingivostomatitis with severe hepatitis in a previously healthy child. J Microbiol Immunol Infect. 2012;45:324-325. 